\title{
Umbilical cord stem cells-
of a new treatment for RA?
}

$\mathrm{M}$ esenchymal stem cells (MSCs) derived from human umbilical cord tissue (UC-MSCs) could be useful in the treatment of rheumatoid arthritis (RA), according to work by Zhang-Huo Li and a team of researchers from Peking University People's Hospital. Their studies in human cells and in an animal model of arthritis demonstrate that UC-MSCs suppress the inflammatory effects of fibroblast-like synoviocytes (FLSs) and induce the expansion of regulatory $\mathrm{T}\left(\mathrm{T}_{\mathrm{REG}}\right)$ cells in vitro, and attenuate collagen-induced arthritis in mice in vivo.

Several lines of research have suggested that stem cells derived from bone marrow (BM-MSCs), the most common source of MSCs, could have immunosuppressive effects, although the effectiveness of these cells in the treatment of RA has been mixed in studies to date. By contrast, little is currently known about the therapeutic potential of UC-MSCs for RA. Despite this lack of knowledge, however, several characteristics of UC-MSCs suggest that they might have several potential advantages over stem cells derived from bone marrow. For instance, the umbilical cord is discarded postnatally and collection of cord tissue is noninvasive. Furthermore, UC-MSCs have a higher proliferation rate and greater self-renewal capacity than MSCs from other sources.

The researchers first isolated MSCs from human umbilical cords after birth and subsequently expanded them in vitro. The expanded cells were morphologically similar to fibroblasts and, functionally, were demonstrably capable of differentiation into osteogenic and adipogenic lineages in culture.

To explore the immunosuppressive potential of UC-MSCs in synovial cells, FLSs were isolated from patients with RA undergoing knee replacement surgery. The proliferation of the isolated FLSs was shown to be sensitive to stimulation by tumor necrosis factor (TNF). In vitro, UC-MSCs inhibited the TNF-induced proliferation of the FLSs in a dose-dependent manner, even when added on the fourth day of a 5-day culture. This inhibitory effect could be abrogated by the addition of 1-MT (an inhibitor of indoleamine 2,3-dioxygenase [IDO]), or antibodies against interleukin (IL)-10 or transforming growth factor (TGF)- $\beta 1$ to the culture, which points to a key role for IDO, IL- 10 and TGF- $\beta 1$ in the observed UC-MSC-mediated inhibition of FLSs.

In cell migration assays, co-culture with UC-MSCs inhibited the invasive behavior of the FLSs in both cell-cell contact and transwell systems. The secretion of matrix metalloproteinase 9, a mediator of the invasive phenotype of FLSs, was also downregulated in both systems. Of note, the expression of the proinflammatory cytokine IL- 6 was inhibited by UC-MSC in the transwell migration assay but not in the cell-cell contact system.

In addition to proliferation and invasive behavior, in vitro studies also demonstrated the capability of UC-MSCs to suppress the inflammatory responses of immune cells from patients with RA. Proliferation of T cells was suppressed by UC-MSCs in a dose-dependent fashion; further experiments determined that this suppressive effect on $\mathrm{T}$ cells relied mainly on TGF- $\beta 1$, nitric oxide and prostaglandin $\mathrm{E}_{2}$. UC-MSCs also inhibited the production of TNF by T cells, and promoted the expansion of $\mathrm{T}_{\mathrm{REG}}$ cells.

Turning to a mouse model of collageninduced arthritis (CIA), Li's team showed that the immunosuppressive effects of UC-MSCs on human T cells and FLSs observed in vitro translate to therapeutic effects in vivo. UC-MSCs infused into mice (after the onset of disease) systemically reduced the severity of CIA. Vehicle alone, dead human FLSs from patients with RA or FLSs from individuals without arthritis had no such effects. Histological examination of sacrificed mice showed that treatment with UC-MSCs prevented tissue damage; by comparison, control mice had pannus formation, inflammatory cell infiltration, severe synovitis and bone erosion.

Consistent with the effects of UC-MSCs on FLSs from RA patients, the inflammatory response in mice with CIA was reduced by treatment with UC-MSCs, including downregulation of TNF production and IL-6, and upregulation of IL-10. Furthermore, T-cell populations shifted from autoreactive type $1 \mathrm{~T}$ helper ( $\mathrm{T}_{\mathrm{H}} 1$ ) cells to IL-4-producing $\mathrm{T}_{\mathrm{H}} 2$ cells. $\mathrm{T}_{\mathrm{REG}}$ cells were also induced, as demonstrated by their increased numbers in the spleen and peripheral blood of treated mice; however, the incidence of delayed-type hypersensitivity responses in treated mice suggests that T-cell priming might have occurred, indicating that a complex mechanism underlies the inflammation-curbing action of UC-MSCs.

Given the apparent immunosuppressive effects and therapeutic potential of UC-MSCs, future research will need to explore both the mechanisms and clinical application of these cells in human diseases including RA.

Sarah Price

Original article Liu, Y. et al. Therapeutic potential of human umbilical cord mesenchymal stem cells in the treatment of rheumatoid arthritis. Arthritis Res. Ther. 12, R210 (2010) 